\title{
Asma agudo severo en niños: ¿Fenotipo diferente?
}

\author{
JAIME LOZANO C.***, LETICIA YÁÑEZ P.**, MICHELANGELO LAPADULA A.**, \\ CARLOS FERNÁNDEZ C.**, ALEX ALCÁNTARA P.**, PILAR RODRÍGUEZ G.**, \\ FELIPE BURGOS F.***, DOLORES ZÚÑIGA P.**** y MÓNICA LAFOURCADE R. *****
}

\section{Severe asthma in children: A different phenotype?}

Background: The magnitude of response to treatment of asthma exacerbations is variable and a significant proportion of them need hospitalization. Objectives: to define the profile of children that were hospitalized for severe asthma and the possible indicators and determinants of their poor responsiveness. Methods: a prospective study in 60 children 4 years or more of age with a search of the ethiology of the exacerbation and a study of the inflammatory profile in sputum. Results: 60 children between 4 and 15 years. 50\% had a previous diagnosis of asthma without regular use of inhaled corticosteroids in two thirds. $40 \%$ had previous admissions for asthma. Etiology of the exacerbation was identified in $52 \%$ with Rhinovirus, human Metapneumovirus, RSV and Mycoplasma pneumoniae as the most frequent agents. Inflammatory profile was determined in 33 children: eosinophilic in $36 \%$, eosinophilic/ neutrophilic in 64\%. Conclusions: Severe asthma with serious exacerbations may be a phenotype whose outstanding aspects in this cohort were: previous hospitalizations, lack of prophylactic treatment, viral infections as frequent trigger, and combined inflammatory cell profile in sputum. mation.

Key words: Severe asthma; children; viral respiratory infections; eosinophilic/neutrophilic inflam-

\section{Resumen}

La magnitud de la respuesta al tratamiento de una exacerbación de asma es variable entre los pacientes y una proporción significativa de ellos debe hospitalizarse. Objetivos: Definir el perfil de los niños que se hospitalizaron por asma grave y los posibles indicadores y determinantes de la respuesta desfavorable al tratamiento. Método: Estudio prospectivo en niños de 4 años o más, con búsqueda etiológica de la exacerbación y estudio de perfil inflamatorio en esputo. Resultados: 60 niños entre 4 y 15 años. El 50\% tenía diagnóstico previo de asma sin uso regular de corticoesteroides inhalados en dos tercios. Hospitalizaciones previas por asma en el 40\%. La etiología de la exacerbación fue identificada en el $52 \%$ siendo los agentes más frecuentes Rhinovirus, Metapneumovius, VRS y Mycoplasma pneumoniae. El perfil inflamatorio fue determinado en 33 niños: eosinofilico en $36 \%$ y eosinofilico/neutrofilico en $64 \%$. Comentario: El asma severa con exacerbaciones graves sería un fenotipo cuyos aspectos destacados en esta cohorte serían: niños con hospitalizaciones previas, falta de tratamiento profiláctico, infección viral como desencadenante frecuente, patrón inflamatorio combinado del esputo y rinitis atópica.

Palabras clave: Asma grave; niños; infección respiratoria viral; inflamación eosinofilica/neutrofilica.

\section{Introducción}

El asma es un síndrome heterogéneo que comprende diferentes fenotipos que se manifiestan con tos, sibilancias, dificultad respiratoria y retracción $\operatorname{costal}^{4,5}$. Es una enfermedad compleja con un componente inflamatorio significativo, caracterizada por episodios de exacerbaciones repetidas y cambios en el patrón inflamatorio de las vías aéreas ${ }^{5}$. Los niños con episodios agudos de asma a diferencia de los adultos presentan un patrón inflamatorio predominantemente eosinofílico en vez de uno neutrofílico ${ }^{4,5,13,14,16}$.

Las exacerbaciones agudas de asma son uno

Clínica Santa María, Santiago. Chile.

* Broncopulmonar.

** Unidad de Paciente Crítico Pediátrico.

*** Kinesiólogo.

**** Tecnólogo médico.

$* * * * *$ Microbiólogo. 
de los problemas frecuentes a enfrentar en los servicios de urgencia ${ }^{3,5}$. El manejo del asma agudo basado en la evidencia incluye $\beta_{2}$ agonistas en todos los pacientes y corticoesteroides por vía sistémica para aquellos con exacerbaciones moderadas y severas ${ }^{1,2,3,7}$. Esta recomendación reduce la tasa de hospitalización en niños y adultos ${ }^{1,2,3}$. Sin embargo, existe marcada heterogeneidad en la magnitud de la respuesta; en la mayoría es favorable pero en otros es insuficiente para evitar la hospitalización por insuficiencia respiratoria. Posiblemente factores tales como edad, sexo, exposición a tabaco, alergenos, infecciones virales, polimorfismo genético y el tipo e intensidad de la inflamación de la vía aérea, modulen la respuesta al corticoesteroide $3,5,6,7,10,11,12,16$. El fenotipo de asma menos conocido es la forma severa de la enfermedad expresada por las exacerbaciones

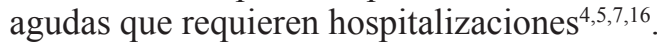

\section{Objetivo primario}

Caracterización de los pacientes pediátricos que se hospitalizan por una exacerbación grave de asma.

\section{Objetivo secundario}

Establecer posibles factores que puedan determinar una deficiente respuesta al tratamiento inicial en el servicio de urgencia

\section{Métodos}

Se realizó un estudio prospectivo que incluyó a niños hospitalizados en la Unidad de Paciente Crítico Pediátrico de la Clínica Santa María (UPCP, CSM) por una exacerbación grave de asma refractaria al tratamiento inicial efectuado en el servicio de urgencia con salbutamol nebulizado y una dosis de metilprednisolona de $1 \mathrm{mg} /$ $\mathrm{kg}$, entre el $1^{\circ}$ de enero de 2008 y el 31 de julio de 2011.

\section{Criterios de inclusión}

Niños mayores de 4 años con los siguientes antecedentes: episodios de sibilancias recurrentes, atopía personal, historia familiar de asma y/o atopía.

\section{Criterios de exclusión}

Cardiopatías congénitas, displasia broncopulmonar, bronquiolitis obliterante, fibrosis quística o inmunodeficiencias. Al ingreso y previa firma del consentimiento informado por los padres, a cada niño se le practicó una evaluación clínica, radiografía $(\mathrm{Rx})$ de tórax, hemograma, detección de IgM en suero para Mycoplasma pneumoniae empleando enzimoinmunoensayo rápido con punto de corte de positividad mayor o igual a 1:64 (InmunoCard ${ }^{\mathrm{R}}$ Mycoplasma) y estudio de Chlamydia pneumoniae y Chlamydia trachomatis mediante detección de $\operatorname{IgG}$ e $\operatorname{IgM}$ en suero por inmunofluorescencia indirecta con técnica de "sandwich" (Chlamydia MIF IgM/IgG Focus Diagnostics) con límite de positividad mayor o igual a 1:10 para IgM y mayor o igual a 1:512 para $\operatorname{IgG}$ (infección aguda). Además se obtuvo con asistencia kinésica, muestras de secreciones respiratorias (aspirado nasofaríngeo o traqueal), para la identificación de los siguientes virus respiratorios: virus respiratorio sincicial (VRS), virus Parainfluenza (PI) 1, 2 y 3, virus Influenza A, B por técnica de Inmunofluorescencia directa (Kit PathoDex ${ }^{\mathrm{R}}$ Respiratory Virus panel, Remel). En el caso de virus Influenza H1N1, se efectuó reacción de polimerasa en cadena (Real Time Influenza A/H1N1, Roche) que detecta gen de matriz de proteína (M2) para Influenza A en combinación con la detección del gen específico de la hemaglutinina HA1. Metapneumovirus humano (hMPV) por reacción de polimerasa en cadena (LightMix ${ }^{\mathrm{R}}$ Kit human MPV Molbiol) que detecta el gen $\mathrm{N}$ de Metapneumovirus, Rhinovirus (RV) por reacción de polimerasa en cadena empleando partidores "in house" para poliproteína (TIB Molbiol) que amplifican segmentos del genoma viral de cepa LZ651 y aislamiento para adenovirus (técnica de shell vial). Otras dos muestras de secreciones respiratorias: una para el estudio de Mycoplasma pneumoniae por reacción de polimerasa en cadena (Light Mix ${ }^{\mathrm{R}}$ Kit Mycoplasma pneumoniae TIB Molbiol Roche) que detecta el gen P1 (proteína citoadhesina) y Bordetella pertussis por inmunofluorescencia directa con anticuerpo monoclonal (Accu-MAb ${ }^{\mathrm{TM}}$ Plus) contra lipopolisacárido específico de Bordetella pertussis y mediante reacción de polimerasa en cadena en tiempo real (LightMix R Kit Bordetella pertussis y parapertussis/TIB Molbiol Roche) que amplifica un segmento de la región IS481 para Bordetella pertussis y de IS1001 para Bordetella parapertussis. La otra muestra fue para el estudio del patrón inflamatorio de la vía aérea (proporción de eosinófilos y neutrófilos). Con la asistencia de un mismo kinesiólogo, se solicitó a los pacientes enjuagar la boca con agua para evitar la contaminación de la muestra de secreciones con células escamosas y luego de inspirar profundamente, toser. El esputo se depositó en un frasco de cultivo. En aquellos niños que no lograron expectorar, la muestra de secreciones fue por aspirado traqueal y se depositó en un kit de aspiración nasotraqueal. A continuación con un aplicador se tomó de la parte más representativa de la muestra una porción que 
fue inmediatamente extendida sobre un portaobjeto seco y limpio. La preparación se fijó en metanol y rotulada fue trasladada al laboratorio de función pulmonar donde fue teñida con tinción de Giemsa. El conteo diferencial lo realizó un tecnólogomédico capacitada, para lo cual debió observar a lo menos 800 células. Fueron consideradas muestras adecuadas para su lectura aquellas con menos de $20 \%$ de células escamosas. Se descartaron aquellas con escasa celularidad y más de $20 \%$ de células escamosas. Para el posterior análisis se consideraron tres patrones inflamatorios: no eosinofílico ( $<2,5 \%$ eosinófilos), eosinofílico $(\geq 2,5 \%$ eosinófilos) y combinado eosinofílico/ neutrofílico $(\geq 2,5 \% \text { eosinófilos y }>54 \% \text { neutrófilos })^{3,5,16}$. El estudio fue aprobado por el comité de ética local.

\section{Resultados}

\section{Pacientes}

Se reclutaron 60 niños entre 4 y 15 años, 54\% hombres. $48 \%$ de los niños presentaron rinitis alérgica. $\mathrm{Al}$ ingreso con diagnóstico establecido de asma bronquial 30 niños; sólo un tercio de ellos en tratamiento regular con corticoesteroides inhalados, en los restantes 20 niños, sus padres habían suspendido el tratamiento sin previa consulta a su médico tratante. Veinticuatro niños (40\%) tenían antecedente de una o más hospitalizaciones por asma de las cuales el $46 \%$ fue en UCI. El episodio actual de asma agudo, fue de rápida instalación: menos de $48 \mathrm{~h}$ de evolución en el $42 \%$ de los casos. En cuatro pacientes existió clara asociación entre una exposición masiva a alergenos (pastos o gatos) y el desarrollo súbito de la exacerbación de asma. Durante la hospitalización todos los pacientes recibieron tratamiento con salbutamol, corticoesteroides por vía sistémica y oxígenoterapia. Necesitaron asistencia ventilatoria no invasiva $29(48 \%)$ e invasiva 3 $(5 \%)$ niños.

\section{Etiologías}

Con el protocolo de estudio propuesto se logró establecer la etiología en 31(52\%) niños (Tabla 1). Rhinovirus fue el principal agente aislado en 13/31 (42\%) seguido por hMPV y Mycoplasma pneumoniae.

\section{Perfil inflamatorio}

Se obtuvo una muestra adecuada para análisis en 33 niños: eosinofílico en $12(36 \%)$ y combinado en 21 (64\%) (Tabla 2). El perfil inflamatorio combinado se asoció a un agente infeccioso en el $62 \%(13 / 21)$ de los casos versus 33\% (4/12) para el perfil inflamatorio eosinofílico (Tabla 3 ).
Tabla 1. Etiologías infecciosas asociadas a la exacerbación severa de asma

\begin{tabular}{|lrc|}
\hline Etiología infecciosa & n & \% \\
Rhinovirus & 11 & 18 \\
Metapneumovirus & 6 & 10 \\
Virus sincicial respiratorio & 4 & 7 \\
Mycoplasma pneumoniae & 4 & 7 \\
Rhinovirus/ VRS & 1 & 1,5 \\
Rhinovirus /Neumococo & 1 & 1,5 \\
H1N1/ Neumococo & 1 & 1,5 \\
H1N1/M. pneumoniae & 1 & 1,5 \\
P. neumoniae & 1 & 1,5 \\
Chlamydia pneumoniae & 1 & 1,5 \\
Total & 31 & 52 \\
\hline
\end{tabular}

Tabla 2. Perfil inflamatorio en secreción respiratoria

\begin{tabular}{|lcc|}
\hline Perfil inflamatorio & n & \% \\
Eosinofílico & 12 & 36 \\
Eosinofílico/Neutrofílico & 21 & 64 \\
Total & 33 & 100 \\
\hline
\end{tabular}

Tabla 3. Relación entre perfil inflamatorio y etiología

\begin{tabular}{|lcc|}
\hline Eosinofílico & $\mathbf{n = 1 2}$ & $\mathbf{\%}$ \\
\hline Sin etiología & 8 & 67 \\
Con etiología & 4 & 33 \\
\hline Eosinofílico/Neutrofílico & $\mathbf{n}=\mathbf{2 1}$ & $\mathbf{\%}$ \\
\hline Sin etiología & 8 & 38 \\
\hline Con etiología & 13 & 62 \\
\hline
\end{tabular}

\section{Discusión}

La administración de dosis repetidas de $\beta_{2}$ agonistas y de corticoesteroides por vía parenteral, fundamentales para el tratamiento de la exacerbación aguda severa de asma, reducen en forma significativa la tasa de hospitalización en escolares y adultos por asma ${ }^{1-3}$. La magnitud de la respuesta a los corticoesteroides en niños es variable ${ }^{3,5-8,16}$. La identificación de los factores que inciden en esta respuesta es difícil, debido a diversos aspectos incluso aquellos relacionados con el diseño de los trabajos recientemente publicados que incluyen poblaciones heterogéneas de niños con asma y bronquiolitis o preescolares con asma inducida sólo por virus u otros niños con fenotipo de asma multigatillante, etc ${ }^{3,6-8}$. Los posibles determinantes de esta respuesta insuficiente 
a corticoesteroides incluyen: edad, sexo, raza, obesidad, exposición a tabaco, fenotipos, factores desencadenantes y genotipos ${ }^{3-8,10-12}$. Así la respuesta inadecuada al tratamiento podría originarse a partir de la interacción entre genes y ambiente para dar como resultado un perfil inflamatorio no eosinofílico de la vía aérea menos respondedor a corticoesteroide ${ }^{3}$. Los objetivos de este trabajo plantean la caracterización de los niños que se hospitalizaron por una exacerbación grave de asma en la UPCP de la CSM debido a la falta de respuesta al tratamiento inicial en el servicio de urgencia y establecer posibles determinantes en el fracaso terapéutico. Para esto la definición de criterios de inclusión y de exclusión permitieron obtener una cohorte homogénea de niños que incluyó a: mayores de 4 años (elimina riesgo de clasificar como asmático un paciente con bronquiolitis), con factores de riesgo para asma ${ }^{3,5,8}$, sin comorbilidad (se excluyeron estos niños para evitar error diagnóstico), con una exacerbación grave de asma (hospitalización por insuficiencia respiratoria) y todos tratados con la misma dosis de corticoesteroide por vía sistémica para descartar mala respuesta por dosis subterapéutica. Aspectos destacados de esta cohorte: alto porcentaje de atópicos, distribución semejante por sexo, episodio agudo de asma de rápida instalación en la mayoría, sorprendentemente con diagnóstico de asma sólo la mitad de los pacientes a pesar de registrar episodios de sibilancias previos, una o más hospitalizaciones e incluso ingreso a Unidades de Paciente Crítico por exacerbaciones graves de asma varios de ellos. Solamente un tercio de los pacientes con diagnóstico de asma establecido previo a esta hospitalización se encontraban con tratamiento regular con corticoesteroide inhalado, en los otros pacientes este fue suspendido por sus padres. Por una parte, la falta de diagnóstico y de tratamiento adecuado y, por otra parte, la no adherencia al tratamiento, predispone a exacerbaciones graves de asma y complicaciones. De lo anterior se desprende la necesidad para el médico especialista de establecer en forma oportuna el diagnóstico de asma, determinar la severidad de la enfermedad, el requerimiento de tratamiento profiláctico y además la incorporación en su práctica clínica diaria de actividades educativas en forma continua al paciente y sus padres para mejorar la adherencia al tratamiento y disminuir de esta manera la morbilidad por asma.

Además de la edad, sexo, raza, exposición a tabaco y exposición masiva a alergenos, existen otros factores que pueden modular la respuesta al corticoesteroide sistémico, entre ellos podemos mencionar los siguientes: infecciones virales, polimorfismo genético y el tipo y magnitud de la respuesta inflamatoria ${ }^{3,5,8-13,15,16}$. La evidencia clínica y experimental sugiere un papel importante de las infecciones respiratorias agudas por virus en las exacerbaciones de asma en niños y adulos ${ }^{3,5,9,10-12,15}$. Las infecciones respiratorias virales son consideradas los gatillantes más frecuentes de exacerbaciones de asma en niños de todas las edades ${ }^{3,5,9,10-12,15}$. La infección viral del epitelio respiratorio produce el reclutamiento y la activación de neutrófilos (degranulación) ${ }^{9}$. En adultos con asma agudo, las infecciones virales, se asocian a hospitalizaciones más prolongadas y a un aumento de los neutrófilos en el esputo, esto sugiere que la inflamación predominantemente neutrofílica de la vía aérea puede ser menos respondedora a corticoesteroide sistémico ${ }^{3,9,12-16}$. En nuestro estudio la aplicación en forma sistemática de un protocolo de estudio etiológico logró establecer un agente infeccioso asociado a la exacerbación de asma en el $52 \%$ de los casos. RV fue el principal agente aislado, seguido por hMPV y VRS y se asociaron preferentemente a un patrón inflamatorio combinado eosinofílico/neutrofílico.

Cada día existe más evidencia que el asma con un fenotipo inflamatorio eosinofílico (exposición a alergenos) tiene mejor respuesta al corticoesteroide sistémico que el asma no eosinofilica , $^{3,5,13,14}$. Una alta proporción de neutrófilos se asocia a infecciones virales, bacterianas, contaminantes ambientales y con la exposición al tabaco ${ }^{3,5,13-16}$. El esputo inducido permite definir el patrón inflamatorio de la vía aérea, tiene buena correlación con el lavado broncoalveolar, pero baja con la biopsia endobronquial ${ }^{5,13,14,16}$ (método invasivo para casos seleccionados). Permite categorizar el patrón inflamatorio en 4 subtipos: eosinofílico, neutrofílico, combinado y paucigranulocítico (eosinófilos y neutrófilos en rango normal) $)^{5,13,14,16}$. La distribución de los fenotipos inflamatorios es diferente entre adultos y niños; en asma estable fenotipo neutrofílico y paucigranulocítico predomina en adultos versus eosinofílico y paucigranulocítico en niños. En asma agudo predomina fenotipo neutrofílico en adultos y eosinofílico en niños. La limitación terapéutica de los corticoesteroides podría deberse a la heterogeneidad de la inflamación entre pacientes y en un mismo paciente en diferentes estados de la enfermedad.

Utilizamos el esputo obtenido con asistencia kinésica en los niños que ingresaron a la UPCP, CSM con una exacerbación aguda de asma, por ser un método seguro, fácil de realizar y no invasivo de monitorizar la inflamación ${ }^{5,13,14,16}$. No se utilizó solución salina para su inducción por riesgo de producir broncoespasmo. Obtuvimos 
una buena muestra para análisis en 33 de los 60 niños reclutados en el estudio. Predominó el fenotipo combinado eosinofílico/neutrofílico sobre el fenotipo eosinofílico. Debemos destacar que el fenotipo combinado se asoció a un agente infeccioso en el $62 \%$ de los casos. De esta forma la respuesta inflamatoria neutrofílica del huésped contra el agente infeccioso se sumaría a la inflamación basal atópica eosinofilica del paciente desencadenando una exacerbación de asma severa con menor respuesta al tratamiento con corticoesteroides.

\section{Comentario}

El fenotipo de asma menos caracterizado es la forma severa de enfermedad que incluye las exacerbaciones graves que requieren hospitalización. Posiblemente la interacción entre genes y el ambiente influyan en el tipo y magnitud de la inflamación de la vía aérea y esta a su vez module la respuesta al corticoesteroide sistémico en niños con asma ${ }^{3}$. El aumento en el porcentaje de neutrófilos en el esputo sugiere un fenotipo inflamatorio predominantemente neutrofílico que puede ser responsable de la respuesta inadecuada al tratamiento. Este estudio representa por una parte un aporte en la caracterización del niño con asma agudo grave (aspectos epidemiológicos, búsqueda etiológica) y, por otra parte, un intento de establecer posibles factores que puedan estar involucrados en la respuesta inadecuada al tratamiento con corticoesteroide sistémico (patrón inflamatorio). Otra posible línea de investigación a desarrollar sobre fenotipificación: niños con asma agudo grave que responden al tratamiento inicial en el servicio de urgencia

\section{Bibliografía}

1.- GLOBAL INITIATIVE FOR ASTHMA (GINA). Global strategy for asthma management and prevention Global Initiative for Asthma (updated 2009). www.ginaasthma. org

2.- BRITISH THORACIC SOCIETY. Scottish Intercollegiate Guidelines Network. British guidelines on the management of asthma - A national clinical guidelines British Thoracic Society. http://www.brit-thoracic.org.uk

3.- DUCHAME F, KRAJINOVIC M. Steroid responsiveness and wheezing phenotypes. Pediatrics Respiratory Reviews 2011; 2: 170-6.

4.- BUSH A, MENZIES-GOW A. Phenotypic differences between pediatric and adult asthma. Am Thorac Soc 2009; 6: 712-9.

5.- HE X, SIMPSON J, WANG F. Inflammatory phenotypes in stable and acute childhood asthma. Pediatric Respira- tory Reviews 2009; 12: 165-9.

6.- HENDERSON J, GRANELL J, SHERIFF A, SIMPSON A, WOODCOCK A, STRACHAN D, et al. Associations of wheezing phenotypes in the first 6 years of life with atopy, lung function and airway responsiveness in midchildhood. Thorax 2008; 63: 974-80.

7.- BRAND PLP, BARALDI E, BISGAARD H, BONER AL, CASTRO-RODRÍGUEZ JA, CUSTOVIC A, et al. Definition, assessment and treatment of wheezing disorders in preschool children: and evidence-based approach. Eur Respir J 2008; 32: 1096-110.

8.- CASTRO-RODRÍGUEZ JA, HOLBERG CI, WRIGHT AL, MARTÍNEZ FD. A clinical index to define risk of asthma in young children with recurrent wheezing. Am J Respir Crit Care Med 2000; 162: 1403-6.

9.- WARK PAB, JOHNSTON SL, MORIC I, SIMPSON JL, HENSLEY MJ, GIBSON PG. Neutrophil degranulation and cell lysis is associated with clinical severity in virus-induced asthma. Eur Respir J 2002; 19: 68-75.

10.- JOHNSTON SL, PATTEMORE PK, SANDERSON G, SMITH S, LAMPE F, JOSEPHS L, et al. Community study of role of viral infection in exacerbations of asthma in 9-11 year old children. Brit Med J 1995; 310 : 1225-9.

11.- JOHNSTON SL, PATTEMORE PK, SANDERSON G, SMITH S, CAMPBELL MJ, JOSEPHS L, et al. The relationship between upper respiratory infections and hospital admissions for asthma: a time-trend analysis. Am J Respir Crit Care Med 1996; 154 (3 Pt 1): 654-60.

12.- HEYMANN PW, PLATTS-MILLS TA, JOHNSTON SL. Role of viral infections, atopy and antiviral immunity in the etiology of wheezing exacerbations among children and young adults. Pediatr Infect Dis J 2005; 24 : s217-22.

13. GIBSON PG, NORZILA MZ, FAKES K, SIMPSON J, HENRY RL. Pattern of airway inflammation and its determinants in children with acute severe asthma. Pediatr Pulmonol 1999; 28: 261-70.

14. PAVORD ID, SHAW DE, GIBSON PD, TAYLOR DR. Inflammometry to assess airway diseases. Lancet 2008; 372: 1017-9.

15. MURRAY CS, POLETTI G, KEBADZE T, MORRIS J, WOODCOCK A, JOHNSTON SL, CUSTOVIC A. Study of modifiable risk factors for asthma exacerbations: virus infection and allergen exposure increase the risk of asthma hospital admissions in children. Thorax 2006; 61: 376-82.

16. BUSH A, SAGLANI S. Management of severe asthma in children. Lancet 2010; 376: 814-25.

Correspondencia a:

Dr. Jaime Lozano C.

Clínica Santa María

Avda. Bellavista 0415, Providencia, Santiago.

E-mail: jlozano@csm.cl 